\title{
Cystatin C is a moderate predictor of acute kidney injury in the early stage of traumatic hemorrhagic shock
}

\author{
SHU CHEN $^{1}$, JING-SONG SHI $^{2}$, XIAOKAITI YIBULAYIN ${ }^{2}$, TIAN-SHAN WU $^{2}$, \\ XIN-WEN YANG ${ }^{2}$, JIE ZHANG ${ }^{2}$ and PAERHATI BAIHETI ${ }^{2}$ \\ ${ }^{1}$ Department of Pathology; ${ }^{2}$ Emergency Center, The First Affiliated Hospital of Xinjiang Medical University, \\ Ürümqi, Xinjiang 830000, P.R. China
}

Received July 27, 2014; Accepted April 1, 2015

DOI: $10.3892 / \mathrm{etm} .2015 .2446$

\begin{abstract}
Patients with traumatic hemorrhagic shock are highly susceptible to the development of acute kidney injury (AKI), but little data are available regarding the changes in cystatin $\mathrm{C}(\mathrm{Cys} C)$ in patients with traumatic hemorrhagic shock. The aim of the present study, therefore, was to investigate whether $\mathrm{Cys} C$ has a higher value than serum creatinine (SCr) and urea for use in monitoring glomerular function in traumatic hemorrhagic shock. Data from a cohort of patients with traumatic hemorrhagic shock, who had been admitted to a trauma center, were collected. Receiver operating characteristic (ROC) curve analysis was used to determine the diagnostic value of serum $\mathrm{CysC}, \mathrm{SCr}$ and urea for the identification of renal dysfunction, and the data were expressed as the area under the curve (AUC). CysC was not significantly affected by gender, age, mechanism of injury or time between injury and arrival at the center in the patients with traumatic hemorrhagic shock. The CysC level of the patients was significantly higher than that of the normal subjects $(1.10 \pm 0.36$ vs. $0.91 \pm 0.34 \mathrm{mg} / \mathrm{l})$; the $\mathrm{SCr}$ and urea levels of the patients were also significantly increased compared with those of the normal subjects. Nonparametric ROC plots of the sensitivity and specificity of $\mathrm{SCr}, \mathrm{CysC}$ and urea for the detection of AKI revealed AUC values of 0.901 [95\% confidence interval (CI), 0.791-1.000], 0.728 (95\% CI, 0.570-0.886) and 0.709 (95\% CI, 0.552-0.865) for $\mathrm{SCr}, \mathrm{CysC}$ and urea, respectively. No significant correlation between mortality and $\mathrm{CysC}, \mathrm{SCr}$ or urea was found. These data indicate that the level of $\mathrm{CysC}$ is significantly increased in the early stage of traumatic hemorrhagic shock and that $\mathrm{CysC}$ can be used as a marker to predict $\mathrm{AKI}$; however, the diagnostic utility of $\mathrm{CysC}$ remains lower than that of $\mathrm{SCr}$ in the early stage of the condition.
\end{abstract}

Correspondence to: Dr Paerhati Baiheti, Emergency Center, The First Affiliated Hospital of Xinjiang Medical University, 137 Liyushan Road, Ürümqi, Xinjiang 830000, P.R. China E-mail: 13090911@qq.com

Key words: cystatin C, acute kidney injury, hemorrhagic shock, trauma

\section{Introduction}

Acute kidney injury (AKI) is a common complication in trauma due to hemorrhage. Despite advances in the provision of hospital emergency procedures, the incidence of AKI is increasing and remains an independent predictor of morbidity and mortality (1-3). An impediment toward improving outcomes has been the continued reliance on outdated and unreliable markers, and there has long been a requirement for a marker that accurately responds to changes in glomerular function

Cystatin $\mathrm{C}(\mathrm{CysC})$ is a $13-\mathrm{kDa}$ endogenous cysteine proteinase inhibitor that plays a major role in the intracellular catabolism of various peptides and proteins (4). CysC is synthesized at a relatively constant rate by all nucleated cells in the human body, and is then released into the plasma. In excess of $99 \%$ of CysC undergoes filtration by the glomeruli, prior to being reabsorbed by proximal renal tubular epithelial cells and catabolized (5). Levels of $\mathrm{CysC}$ can therefore be used to measure glomerular function. Furthermore, since an increased presence of $\mathrm{Cys} C$ in the urine may indicate damage to the tubular epithelium, the protein has been proposed as an additional urine biomarker for AKI (6).

Estimates for the glomerular filtration rate (GFR) based on measurements of $\mathrm{Cys} C$ have been suggested to be superior to those based on serum creatinine ( $\mathrm{SCr}$ ) in selected patient populations, such as the elderly and children, as well as transplant, cirrhotic and hypertensive patients (7-10); however, the results in cardiac surgery and critically ill patients are inconclusive (11-15). Several studies have also shown that serum CysC has a similar, and not necessarily superior, value to $\mathrm{SCr}$ for the detection of AKI in the early stage of the disease. Patients with traumatic hemorrhagic shock are highly susceptible to the development of AKI, but little data are available regarding the changes in CysC in patients with traumatic hemorrhagic shock. The aim of the present study, therefore, was to investigate whether $\mathrm{CysC}$ has a higher value than $\mathrm{SCr}$ and urea for use in monitoring glomerular function in traumatic hemorrhagic shock.

\section{Patients and methods}

Subject population. For the experimental group, diagnostic serum samples were obtained from 57 patients who 
were diagnosed with traumatic hemorrhagic shock in the Emergency Center of the First Affiliated Hospital of Xinjiang Medical University (Ürümqi, China). All patients admitted to the Emergency Center between July 1, 2012 and July 1, 2013 were included in the cohort, which comprised 41 men and 16 women, aged $34.49 \pm 17.28$ years. A total of 57 normal serum samples were randomly collected from individuals during the same period to form a control group. The control group also consisted of 41 men and 16 women, aged $36.24 \pm 15.22$ years. All of the patients and volunteers provided written informed consent, and the study was approved by the University and Institutional Review Board (First Affiliated Hospital of Xinjiang Medical University). No significant differences in gender and age were found between the groups. The initial values of $\mathrm{CysC}, \mathrm{SCr}$ and urea in the patients with trauma and normal subjects were measured.

Definitions. For inclusion in the experimental group, patients had to exhibit at least one of the following injuries: Fracture to the pelvis, two or more long bones or multiple ribs; pulmonary contusion; or major blunt or penetrating trauma to the extremities or torso (16). In addition, patients had to have two systolic blood pressure measurements of $<90 \mathrm{mmHg}$ or a base deficit of $\geq 6 \mathrm{mEq} / 1$ within $60 \mathrm{~min}$ of arrival at the Emergency Center. All of the samples were collected within the first $8 \mathrm{~h}$ after trauma. Patients who succumbed prior to reaching the Emergency Center were excluded from the study.

Normal subjects were defined as those without the following conditions: High blood pressure; diabetes; liver, kidney, heart and infectious diseases; acute infection within the past month; and any malignancy. AKI was defined as an $\mathrm{SCr}$ increase of $\geq 50 \%$ or $\geq 27 \mathrm{mmol} / 1$ within $48 \mathrm{~h}$. SCr was measured using the isotope dilution mass spectrometry traceable enzymatic method (Roche Diagnostics, Mannheim, Germany), urea was measured using an enzymatic kit (Roche) and CysC was measured by nephrometric assay (Dade Behring, Pittsburgh, PA, USA) according to the manufacturer's instructions.

Statistical analysis. Data are expressed as the mean \pm standard deviation and were analyzed using SPSS 17.0 statistical software (SPSS, Inc., Chicago, IL, USA). Comparisons between groups were made using the t-test or one-way analysis of variance for continuous variables and either the Fisher's exact or $\chi^{2}$ test for categorical variables. Correlation analysis for quantitative data was performed using Pearson's test. To investigate the diagnostic value of serum $\mathrm{CysC}, \mathrm{SCr}$ and urea as predictors of AKI, receiver operating characteristic (ROC) curve analysis was performed; these data are expressed as the area under the curve (AUC) and 95\% confidence interval (CI). Multivariate logistic regression analysis was used to investigate the association between these values and mortality. $\mathrm{P}<0.05$ was considered to indicate a statistically significant difference.

\section{Results}

Characteristics of the study subjects. Table I shows the characteristics of the experimental group subjects. The mean CysC value of the female patients was lower compared with the male patients $(0.96 \pm 0.22$ vs. $1.16 \pm 0.39 \mathrm{mg} / 1$, respectively), but no significant difference was found $(\mathrm{P}>0.05)$. The $\mathrm{CysC}$ level was

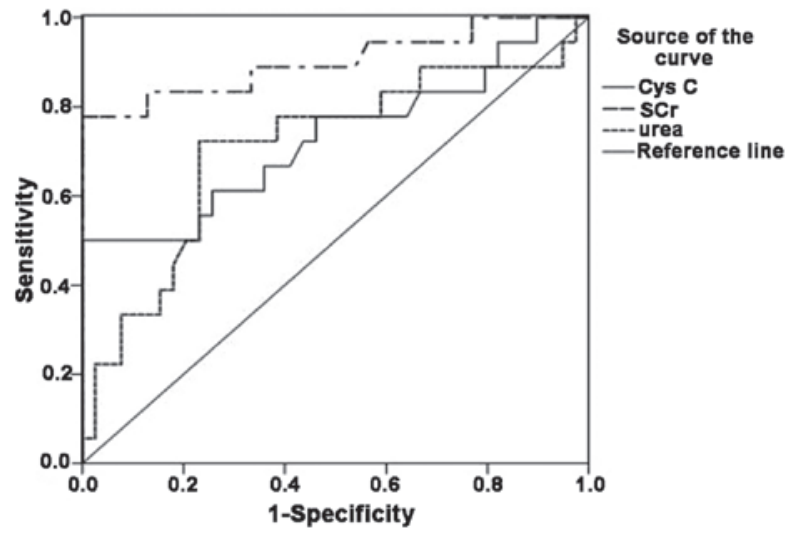

Figure 1. Receiver operating characteristic curve of CysC, $\mathrm{SCr}$ and urea. The area under the curve for the three indicators was as follows: $\mathrm{SCr}, 0.901$ (95\% CI, 0.791-1.000); CysC, 0.728 (95\% CI, 0.570-0.886); and urea, 0.709 (95\% CI, 0.552-0.865). CysC, cystatin C; $\mathrm{SCr}$, serum creatinine; $\mathrm{CI}$, confidence interval.

not significantly affected by age, mechanism of injury or time between injury and arrival at the Emergency Center.

The CysC level of the patients with traumatic hemorrhagic shock was significantly higher than that of the normal subjects $(1.10 \pm 0.36$ vs. $0.91 \pm 0.34 \mathrm{mg} / \mathrm{l})$. The $\mathrm{SCr}$ and urea levels in the experimental group were also significantly increased compared with those in the control group (Table II).

Association between AKI and SCr, serum CysC and urea. A total of 18 of the 57 patients with traumatic hemorrhagic shock enrolled in the study had AKI (SCr increase of $\geq 50 \%$ or $\geq 27 \mathrm{mmol} / \mathrm{l}$ within $48 \mathrm{~h}$ ). Fourteen of these 18 patients with AKI had elevated $\mathrm{SCr}$ concentrations in the first $6 \mathrm{~h}$ of trauma, whereas 13 had elevated serum CysC levels at that time. Only 10 of the patients had elevated urea levels.

Nonparametric ROC plots of the sensitivity and specificity of $\mathrm{SCr}, \mathrm{CysC}$ and urea for the detection of $\mathrm{AKI}$ are shown in Fig. 1. The AUCs for SCr, CysC and urea were 0.901 (95\% CI, $0.791-1.000), 0.728$ (95\% CI, 0.570-0.886) and 0.709 (95\% CI, $0.552-0.865$ ), respectively (Table III and Fig. 1).

Correlation analysis for serum indicators. Pearson correlation analysis was used to evaluate the correlations among the quantitative data. A significant correlation was found between any two of $\mathrm{CysC}, \mathrm{SCr}$ and urea $(\mathrm{P}<0.01$; Table IV).

Association between serum indicators and mortality. Six of the 57 patients enrolled in the study succumbed. Using multivariate logistic regression analysis, the association between mortality and the levels of $\mathrm{CysC}, \mathrm{SCr}$ and urea was determined in the study population. The three variables were excluded from the multivariate model, and no significant correlation was found between mortality and the levels of $\mathrm{CysC}, \mathrm{SCr}$ and urea.

\section{Discussion}

AKI is commonly encountered in cases of traumatic hemorrhagic shock. The development of AKI is associated with poorer clinical outcomes, including a prolonged period of 
Table I. Characteristics of the study subjects.

\begin{tabular}{lcc}
\hline Clinical parameter & $\mathrm{N}$ & CysC (mg/l) \\
\hline Gender & 41 & $1.16 \pm 0.39$ \\
Male & 16 & $0.96 \pm 0.22$ \\
Female & & \\
Age (years) & 10 & $1.04 \pm 0.23$ \\
0 to $<20$ & 23 & $0.97 \pm 0.24$ \\
20 to $<40$ & 20 & $1.26 \pm 0.48$ \\
40 to $<60$ & 4 & $1.27 \pm 0.33$ \\
$\geq 60$ & & \\
Mechanism of injury & 41 & $1.09 \pm 0.38$ \\
Blunt & 6 & $1.17 \pm 0.35$ \\
Penetrating & 10 & $1.12 \pm 0.34$ \\
Both & & \\
Time between injury and arrival (h) & 28 & $1.07 \pm 0.38$ \\
0 to $<5$ & 29 & $1.13 \pm 0.35$ \\
$5-8$ & & 0.865 \\
\hline
\end{tabular}

Table II. Comparative analysis of levels of $\mathrm{CysC}, \mathrm{SCr}$ and urea between patients with traumatic hemorrhagic shock and normal subjects.

\begin{tabular}{lccc}
\hline Parameter & Experimental group & Control group & P-value \\
\hline CysC $(\mathrm{mg} / \mathrm{l})$ & $1.10 \pm 0.36$ & $0.91 \pm 0.34$ & 0.004 \\
$\mathrm{SCr}(\mu \mathrm{mol} / \mathrm{l})$ & $81.93 \pm 41.35$ & $58.15 \pm 16.13$ & $<0.001$ \\
Urea $(\mathrm{mmol} / \mathrm{l})$ & $5.06 \pm 1.95$ & $3.89 \pm 1.07$ & $<0.001$ \\
\hline
\end{tabular}

CysC, cystatin C; SCr, serum creatinine.

Table III. Area under the curve values for $\mathrm{CysC}, \mathrm{SCr}$ and urea.

\begin{tabular}{lrrr}
\hline Variable & Area & P-value & \multicolumn{1}{c}{$95 \%$ CI } \\
\hline CysC & 0.728 & 0.006 & $0.570-0.886$ \\
SCr & 0.901 & $<0.001$ & $0.791-1.000$ \\
Urea & 0.709 & 0.012 & $0.552-0.865$
\end{tabular}

CysC, cystatin C; SCr, serum creatinine; CI, confidence interval.

hospitalization, a dependence upon renal replacement therapy, the development of chronic kidney disease and an increased risk of mortality $(2,3,16)$.

Traditionally, the diagnosis of AKI has been dependent upon the use of surrogate markers to detect abnormalities in kidney function, such as elevations in SCr or urea. Although considerable research has been performed in this area, the diagnosis of AKI still relies on the same surrogates of kidney function, which often are poor markers of early kidney injury; therefore, the identification of novel kidney-specific biomarkers for the early detection of AKI and kidney dysfunction is considered to be a priority.
Table IV. Pearson correlation analysis of CysC, $\mathrm{SCr}$ and urea.

\begin{tabular}{llll}
\hline Parameter & CysC & SCr & Urea \\
\hline CysC & & & \\
Pearson correlation & 1.000 & 0.457 & 0.369 \\
P-value & & $0.000^{\mathrm{a}}$ & $0.005^{\mathrm{a}}$ \\
SCr & & & \\
Pearson correlation & 0.457 & 1.000 & 0.439 \\
P-value & $0.000^{\mathrm{a}}$ & & $0.001^{\mathrm{a}}$ \\
Urea & & & \\
$\quad$ Pearson correlation & 0.369 & 0.439 & 1.000 \\
P-value & $0.005^{\mathrm{a}}$ & $0.001^{\mathrm{a}}$ &
\end{tabular}

${ }^{\mathrm{a}} \mathrm{P}<0.01$. CysC, cystatin $\mathrm{C}$; SCr, serum creatinine.

CysC is easy to measure and has been regarded as an ideal marker of kidney function (17). Data suggest that CysC is modified by gender, age, muscle mass, obesity, smoking status, thyroid function, inflammation and malignancy (18). In the present study, the levels of $\mathrm{CysC}$ detected were highest in male patients and patients aged $>40$ years; however, the level 
of CysC was not significantly associated with gender, age, mechanism of injury or time between injury and arrival at the Emergency Center in the patients with traumatic hemorrhagic shock.

Normal serum samples, collected during the same period as that during which the patient samples were collected, were used to form the control group; the two groups were matched in terms of gender and age. The early stage of traumatic hemorrhagic shock is associated with a decline in kidney function and, in the present study, it was found that the levels of CysC, $\mathrm{SCr}$ and urea in the patients with traumatic hemorrhagic shock were significantly higher than those in the normal subjects.

The GFR in individuals suffering from critical trauma can change rapidly, and changes in the $\mathrm{SCr}$ can be apparent for several days until the stabilization phase is reached (19). In the present study, 18 out of the 57 (31.6\%) enrolled subjects developed AKI and exhibited high SCr levels within $48 \mathrm{~h}$. This result was similar to findings in other studies $(20,21)$. The diagnostic utility of CysC (AUC, 0.728) observed in the present study was similar to that of urea (AUC, 0.709); however, $\mathrm{SCr}$ (AUC, 0.901) remained the most suitable marker for the prediction of AKI. Although previous reports have suggested that $\mathrm{CysC}$ represents a better marker than $\mathrm{SCr}$ in several fields, the present findings have shown the diagnostic utility of CysC to be lower than that of $\mathrm{SCr}$ in the early stage of traumatic hemorrhagic shock.

A strong correlation between any two of $\mathrm{CysC}, \mathrm{SCr}$ and urea was found in this study. This result was similar to findings in other studies (22-24). None of the serum indicators, however, showed a significant correlation with mortality. Previous reports have found that approximately one-third of patients with traumatic hemorrhagic shock develop AKI (25-27). Hospital care should be adjusted accordingly, but the incidence of AKI cannot be use to predict mortality in cases of traumatic hemorrhagic shock.

In conclusion, the present data indicate that $\mathrm{CysC}$ levels are increased significantly in the early stage of traumatic hemorrhagic shock. CysC can be used as a marker to predict AKI, but the diagnostic utility of $\mathrm{Cys} C$ remains lower than that of $\mathrm{SCr}$ in the early stage of traumatic hemorrhagic shock.

\section{References}

1. de Abreu KL, Silva Júnior GB, Barreto AG, et al: Acute kidney injury after trauma: Prevalence, clinical characteristics and RIFLE classification. Indian J Crit Care Med 14: 121-128, 2010.

2. Bihorac A, Delano MJ, Schold JD, et al: Incidence, clinical predictors, genomics and outcome of acute kidney injury among trauma patients. Ann Surg 252: 158-165, 2010.

3. Costantini TW, Fraga G, Fortlage D, et al: Redefining renal dysfunction in trauma: Implementation of the Acute Kidney Injury Network staging system. J Trauma 67: 283-288, 2009.

4. Goldstein SL: A novel use for novel acute kidney injury biomarkers: Fenoldopam's effect on neutrophil gelatinase-associated lipocalin and cystatin C. Crit Care 15: 177, 2011.

5. Bagshaw SM and Bellomo R: Cystatin C in acute kidney injury. Curr Opin Crit Care 16: 533-539, 2010.
6. Soto K, Coelho S, Rodrigues B, et al: Cystatin C as a marker of acute kidney injury in the emergency department. Clin J Am Soc Nephrol 5: 1745-1754, 2010.

7. Gerhardt T, Pöge U, Stoffel-Wagner B, et al: Estimation of glomerular filtration rates after orthotopic liver transplantation: Evaluation of cystatin C-based equations. Liver Transpl 12: 1667-1672, 2006.

8. Pöge U, Gerhardt T, Stoffel-Wagner B, et al: Calculation of glomerular filtration rate based on cystatin $\mathrm{C}$ in cirrhotic patients. Nephrol Dial Transplant 21: 660-664, 2006.

9. Pöge U, Gerhardt T, Stoffel-Wagner B, et al: Cystatin C-based calculation of glomerular filtration rate in kidney transplant recipients. Kidney Int 70: 204-210, 2006.

10. Rodilla E, Costa JA, Pérez Lahiguera F, González C, Miralles A and Pascual JM: Cystatin C and other cardiovascular markers in hypertension. Med Clin (Barc) 130: 1-5, 2008 (In Spanish).

11. Haase M, Bellomo R, Devarajan P, et al: Novel biomarkers early predict the severity of acute kidney injury after cardiac surgery in adults. Ann Thorac Surg 88: 124-130, 2009.

12. Koyner JL, Bennett MR, Worcester EM, et al: Urinary cystatin C as an early biomarker of acute kidney injury following adult cardiothoracic surgery. Kidney Int 74: 1059-1069, 2008.

13. Ristikankare A, Pöyhiä R, Kuitunen A, et al: Serum cystatin C in elderly cardiac surgery patients. Ann Thorac Surg 89: 689-694, 2010.

14. Nejat M, Pickering JW, Walker RJ and Endre ZH: Rapid detection of acute kidney injury by plasma cystatin $\mathrm{C}$ in the intensive care unit. Nephrol Dial Transplant 25: 3283-3289, 2010.

15. Perianayagam MC, Seabra VF, Tighiouart $\mathrm{H}$, Liangos O and Jaber BL: Serum cystatin C for prediction of dialysis requirement or death in acute kidney injury: A comparative study. Am J Kidney Dis 54: 1025-1033, 2009.

16. Morrison CA, Carrick MM, Norman MA, et al: Hypotensive resuscitation strategy reduces transfusion requirements and severe postoperative coagulopathy in trauma patients with hemorrhagic shock: Preliminary results of a randomized controlled trial. J Trauma 70: 652-663, 2011.

17. Ortuno-Anderiz F, Cabello-Clotet N, Vidart-Simon N, et al: Cystatin $\mathrm{C}$ as an early marker of acute kidney injury in septic shock. Rev Clin Esp 215: 83-90, 2015.

18. Bagshaw SM and Bellomo R: Cystatin $\mathrm{C}$ in acute kidney injury. Curr Opin Crit Care 16: 533-539, 2010.

19. Bihorac A, Baslanti TO, Cuenca AG, et al: Acute kidney injury is associated with early cytokine changes after trauma. J Trauma Acute Care Surg 74: 1005-1013, 2013.

20. de Abreu KL, Silva Junior GB, Barreto AG, et al: Acute kidney injury after trauma: Prevalence, clinical characteristics and RIFLE classification. Indian J Crit Care Med 14: 121-128, 2010.

21. Talving P, Karamanos E, Skiada D, Lam L, Teixeira PG, Inaba K, Johnson J and Demetriades D: Relationship of creatine kinase elevation and acute kidney injury in pediatric trauma patients. J Trauma Acute Care Surg 74: 912-916, 2013

22. Hei ZQ, Li XY, Shen N, et al: Prognostic values of serum cystatin $\mathrm{C}$ and beta 2 microglobulin, urinary beta 2 microglobulin and $\mathrm{N}$-acetyl-beta-D-glucosaminidase in early acute renal failure after liver transplantation. Chin Med J 121: 1251-1256, 2008.

23. Liu J: Evaluation of serum cystatin $\mathrm{C}$ for diagnosis of acute rejection after renal transplantation. Transplant Proc 44: 1250-1253, 2012.

24. Lagos-Arevalo P, Palijan A, Vertullo L, et al: Cystatin C in acute kidney injury diagnosis: early biomarker or alternative to serum creatinine? Pediatr Nephrol 30: 665-676, 2015.

25. Gomes E, Antunes R, Dias C, et al: Acute kidney injury in severe trauma assessed by RIFLE criteria: A common feature without implications on mortality? Scand J Trauma Resusc Emerg Med 18: 1, 2010.

26. Podoll AS, Kozar R, Holcomb JB, et al: Incidence and outcome of early acute kidney injury in critically-ill trauma patients. PLoS One 8: e77376, 2013.

27. Skinner DL, Hardcastle TC, Rodseth RN, et al: The incidence and outcomes of acute kidney injury amongst patients admitted to a level I trauma unit. Injury 45: 259-264, 2014. 